Published in final edited form as:

Inflamm Bowel Dis. 2015 February ; 21(2): 345-352. doi:10.1097/MIB.0000000000000270.

\title{
Body Image Dissatisfaction in Patients with Inflammatory Bowel Disease
}

\author{
Sumona Saha, M.D., M.S., \\ Division of Gastroenterology and Hepatology, University of Wisconsin School of Medicine and \\ Public Health

\section{Ying-Qi Zhao, Ph.D.,} \\ Department of Biostatics and Medical Informatics, University of Wisconsin School of Medicine \\ and Public Health
}

\section{Samir A. Shah, M.D.,}

Division of Gastroenterology, Warren Alpert School of Medicine at Brown University

\section{Silvia Degli Esposti, M.D.,}

Division of Gastroenterology, Warren Alpert School of Medicine at Brown University

\section{Sheldon Lidofsky, M.D.,}

Division of Gastroenterology, Warren Alpert School of Medicine at Brown University

Jason Shapiro, M.D.,

Division of Pediatric Gastroenterology, Warren Alpert School of Medicine at Brown University

Neil LeLeiko, M.D., Ph.D.,

Division of Pediatric Gastroenterology, Warren Alpert School of Medicine at Brown University

Renee Bright, M.S.,

Department of Pediatric Gastroenterology, Rhode Island Hospital

Meaghan Law,

Department of Pediatric Gastroenterology, Rhode Island Hospital

\section{Heather Moniz,}

Department of Pediatric Gastroenterology, Rhode Island Hospital

Zahid Samad, M.D., M.P.H., M.B.A.,

Centers for Disease Control and Prevention, National Center for Chronic Disease Prevention and Health Promotion

\section{Marjorie Merrick, M.A., and}

Crohn's \& Colitis Foundation of America

Bruce E. Sands, M.D., M.S.

Dr. Henry D. Janowitz Division of Gastroenterology, Icahn School of Medicine at Mount Sinai

Correspondence: Please address correspondence regarding this manuscript to: Sumona Saha, M.D., M.S., Division of

Gastroenterology and Hepatology, University of Wisconsin School of Medicine and Public Health, UW Medical Foundation

Centennial Building, 1685 Highland Avenue, Rm 4224, Madison, WI 53705, Phone: 608-263-1995, Fax: 608-265-5677,

ssaha@medicine.wisc.edu. 


\section{Abstract}

Background-Despite the fact that the inflammatory bowel diseases (IBD) and their treatments may affect physical appearance, the effect of IBD on body image is poorly understood. The aims of this study were to determine whether body image dissatisfaction (BID) changes over time in patients with IBD and to examine the demographic and disease-related variables associated with decreased body image.

Methods-Adults aged 18 and above in the Ocean State Crohn's and Colitis Area Registry with at least 2 years of follow-up were eligible for this study. All patients were enrolled within 6 months of IBD diagnosis and followed prospectively. BID was assessed using a modified version of the Adapted Satisfaction With Appearance (ASWAP) questionnaire. Total ASWAP scores and 2 sub-scores were calculated. To assess for changes over time, general linear models for correlated data were used for continuous outcomes and generalized estimating equations were used for discrete outcomes.

Results-Two hundred seventy-four patients were studied. BID was found to be stable over time among men and women with IBD despite overall improvements in disease activity. No differences were found in BID according to IBD subtype. Female gender, greater disease activity, higher symptom burden, longer duration of steroid use, dermatologic and musculoskeletal manifestations of IBD, and ileocolonic disease location among patients with Crohn's disease were associated with greater BID. Greater BID was associated with lower health-related quality of life (HRQOL).

Conclusions-BID remains stable in an incident cohort of IBD despite improved disease activity and is associated with lower HRQOL

\section{Introduction}

Body image encompasses body-related self-perceptions and self-attitudes, such as body (dis-)satisfaction. ${ }^{1}$ The subjective evaluation of one's appearance is a central aspect of body image. ${ }^{2}$ Negative body image is associated with depressive symptoms, social anxiety and avoidance of social situations, low self-esteem, disordered eating, and other forms of poor self-care. ${ }^{3,4}$ In addition, in adults it has been linked to decreased sexual satisfaction and impairments in interpersonal relationships. ${ }^{1,5}$

Body image dissatisfaction (BID) has been evaluated in adults with medical disorders in which appearance-related changes and disfigurements are readily visible and interpersonally salient. In such populations it has been shown that appearance-related changes can pose significant challenges to the preservation of positive self-esteem and body image. ${ }^{6}$ Among patients with inflammatory bowel diseases (IBD), BID has been poorly evaluated. A singlecenter study by Muller et al. suggested that as many as two-thirds of patients with IBD have impaired body image, however, studies validating these findings using standardized measures are lacking. ${ }^{7}$

Although IBD is a disorder of the gastrointestinal tract, it is well known that both ulcerative colitis (UC) and Crohn's disease (CD) as well as their treatments can dramatically change the functioning and appearance of the body, which in turn may alter patients' body image and psychosocial well-being. Patients with active disease may experience changes in 
appearance due to weight loss, hair loss, or one of several cutaneous manifestations of IBD. ${ }^{8,9}$ In addition, patients with CD may develop disfiguring fistulas to the skin and perineum. Even patient with disease in remission may experience changes in physical appearance due to the potential of corticosteroids to cause weight gain, lipodystrophy and acne, azathioprine and 6-mercaptopurine (6-MP) to cause photosensitivity, methotrexate to cause hair loss, and the tumor necrosis factor alpha (TNF)-a inhibitors to cause psoriasis. ${ }^{10-13}$ Furthermore, patients who undergo surgical management may struggle with the body-altering effects of scars and stomas.

The primary purpose of this study was to determine the prevalence of BID and its risk factors in adults with newly diagnosed IBD and the changes that occur in BID over time. A secondary goal was to examine the effect of BID on health-related quality of life (HRQOL) in patients with IBD.

\section{Methods}

\section{Participants and Procedures}

Patients aged 18 and above enrolled in the Ocean State Crohn's and Colitis Area Registry (OSCCAR) with a minimum of 2 years of follow-up were eligible for this study. OSCCAR is a community-based incident cohort of IBD based in the state of Rhode Island that was established in 2008. Subjects enrolled in OSCCAR have UC, CD, or inflammatory bowel disease undetermined (IBDU) based on endoscopy, radiology, and/or pathology. Eligible subjects must be enrolled within 6 months of diagnosis, with a median time to enrollment for adults of approximately 90 days from the date of diagnosis.

Subjects in OSCCAR undergo assessment of symptoms using a comprehensive symptom inventory, disease activity as measured by the Harvey-Bradshaw Index (HBI ${ }^{14}$ for subjects with CD (range 0 to $\infty$ ) or Simple Clinical Colitis Index (SCCAI) ${ }^{15}$ (range 0 to 19) for subjects with UC or IBDU, medication use, surgical history, general and disease-specific HRQOL as measured by Inflammatory Bowel Disease Questionnaire (IBDQ) ${ }^{16}$ (range 32 to 224 , with higher scores indicating better quality of life) at the time enrollment and then every 6 to 12 months, depending on the outcome being measured. Disease location is recorded according to the Montreal Classification. ${ }^{17}$

To assess BID a modified version of the Adapted Satisfaction with Appearance (ASWAP) scale was administered. ${ }^{18}$ The ASWAP is an adaption of the Satisfaction With Appearance (SWAP) scale. SWAP and ASWAP measure non-weight-related BID at multiple body sites (e.g. face, extremities, chest) and social discomfort related to appearance. SWAP was developed and tested on burn victims where it was found to have good internal consistency and moderate test-retest reliability. ${ }^{19}$ ASWAP was administered to participants with scleroderma to evaluate its psychometric integrity and its validity for use in a different medical population that experiences changes in appearance. ${ }^{18}$ The ASWAP re-introduces a fifteenth item that was excluded from the final version of the SWAP ("My appearance makes others feel uncomfortable") and changed items that included the word "burn" to "illness". Principal component analysis of the ASWAP revealed two factors, Subjective 
Dissatisfaction and Perceived Social Impact, rather than the four found in burn victims. We replaced an item addressing $=$ satisfaction with scalp with an item about the abdomen .

On the SWAP and ASWAP, subjects are asked to rate the degree to which each item accurately reflects their thoughts and feelings about their appearance. Ratings are made on a 7-point scale ranging from 1 (strongly disagree) to 7 (strongly agree). We calculated total modified ASWAP scores by subtracting 1 from each item in order to anchor queries at 0 and then adding items in the subscales. According to the initial description of the measure, questions corresponding to items 4-11 in the SWAP were reverse scored such that higher scores reflect greater dissatisfaction or distress.${ }^{19}$ Two sub-scores (Perceived Social Impact and Subjective Dissatisfaction) were calculated for each subject at baseline and at each follow-up time point.

OSCCAR has been approved by the Institutional Review Boards at Lifespan/Rhode Island Hospital, Massachusetts General Hospital, Icahn School of Medicine at Mount Sinai, and the University of Wisconsin School of Medicine and Public Health.

\section{Data Analysis}

We described the demographic characteristics, IBD subtype (CD, UC or IBDU), disease characteristics, medication use, IBD activity as assessed by the HBI and the SCCAI, symptoms, surgical history, and HRQOL as measured by the IBDQ of the cohort at baseline and follow-up. To assess changes in BID according to these factors, a general linear model for correlated data was used.

We used logistic regression to calculate the propensity score of steroids usage. At each time point, the predictors for the model included age at enrollment, disease activity score, total number of symptoms, indicators for other medication usage at current time points and indicators for steroids usage at previous time points. At baseline, we used a weighted regression analysis, with weight being the inverse probability of using steroids, to assess the steroids effect. Weighting was done to account for dissimilar characteristics between patients who do and do not take steroids, and achieve a pseudo-sample where the outcome is not confounded by the variables in the propensity score. ${ }^{20}$ Due to inconsistences in patient recall with regards to steroid dosing, we used duration of steroid use as a surrogate measure of steroid exposure. To evaluate the effect of the duration of steroid exposure on ASWAP scores we used marginal structural models. ${ }^{21}$ The current analysis is valid under the assumption of data "missing at random". 22

\section{Results}

Study Population-Of 389 eligible subjects, 274 (70.4\%) participated in this study (145 CD, 129 UC/IBDU). Respondents were $61.7 \%$ female. The mean age (SD) of the subjects at diagnosis was 40.6 (16.8) and mean (SD) body mass index (BMI) was 27.4 (7.3). With regards to baseline disease activity, mean (SD) HBI score for subjects with CD was 3.2 (3.5) and mean (SD) SCCAI score for subjects with UC and IBDU was 2.7 (2.6) across three years. Further demographic information and disease characteristics of the population at baseline are summarized in Tables 1 and 2 . 
Body Image Dissatisfaction-The modified ASWAP scores for subjects stratified by sex and adjusted for age and marital status at baseline and follow-up are summarized in Table 3. After adjusting for age and marital status, we did not find significant improvement in BID either by total ASWAP score or by either sub-scores, from baseline to year 2 of follow-up ( $\mathrm{p}=0.08$ ). BID remained stable over time for both males and females.

When comparing total ASWAP scores and sub-scores between sexes we found all scores at each time point to be significantly different between males and females $(\mathrm{p}<0.0001)$. Total ASWAP scores were 6.78 (SE 1.58) higher among females compared to males while Perceived Social Impact scores were 2.12 (SE 0.81) higher and Subjective Dissatisfaction scores were 4.58 (SE 0.92) higher, indicating greater BID among females compared to males.

After adjusting for age, marital status, and gender we found no association between BID and IBD subtype. Symptom burden, however, was associated with all BID scores (i.e. total ASWAP score and both sub-domain scores) wherein subjects with a greater number of symptoms had higher BID scores ( $\mathrm{p}<0.0001)$. As expected, given that the ASWAP scale is a measure of non-weight related BID, BMI was not significantly associated with BID by any measures after adjusting for multiple comparisons (Table 4).

Disease location: With regards to disease location, we found significant differences in ASWAP scores among patients with CD wherein patients with ileocolonic disease (L3) had the highest BID scores while subjects with colonic disease (L2) had the lowest scores (Table 4). No difference was found between patients with isolated ileal disease (L1) and patients with either L2 or L3 disease. Within the UC cohort, we found no significant difference in ASWAP scores by disease location. Compared to subjects without perianal disease, total ASWAP scores were estimated to be 3.60 (SE 4.76) higher for subjects with CD with perianal involvement after adjusting for age, gender, and marital status. This difference, however, was not significant. This may be due to insufficient power as only 8 subjects with $\mathrm{CD}$ had documented perianal disease at last follow-up.

Extraintestinal manifestations: When comparing total ASWAP scores and ASWAP subscores between subjects with an extraintestinal manifestation (EIM) of IBD and without, we found all scores at each time point to be significantly different between the two groups ( $p<0.0001$ (Table 4) Total ASWAP scores were 4.15 (SE 1.10) higher in subjects with an EIM compared to subjects without after adjusting for age, gender, and marital status. Perceived social impact and subjective dissatisfaction scores were 1.76 (SE 0.59) and 3.15 (SE 0.71) higher in subjects with an EIM compared with subjects without.

Regarding specific EIMs, we found that over time, presence of musculoskeletal $(\mathrm{p}=0.0001)$ and dermatologic manifestations $(\mathrm{p}=0.03)$ were significantly associated with total ASWAP scores and that presence of ocular manifestation was not after adjusting for multiple confounders.

Surgery: Only 6 patients had a history of an IBD-related surgery at baseline and only 17 had undergone IBD-related surgery by the end of follow-up. Comparing total ASWAP 
scores between subjects who had and who had not undergone surgery at baseline and by the end of follow-up did not reveal a significant difference.

Medication usage: We analyzed the effect of IBD medication use on BID and found steroid use (current or recent) was significantly associated with greater BID by all measures at baseline and all subsequent time points $(\mathrm{p}=0.02)$ (Table 4$)$. When comparing the effect of steroid use on BID by gender, we found that the changes in BID scores with steroid exposure were significantly different between males and females, wherein the magnitude of change in total ASWAP scores was greater in females compared to males $(\mathrm{p}=0.04)$. The mean difference in total ASWAP scores was 4.43 (SE 2.12) over all years.

Using logistic regression and after controlling for propensity for baseline steroid use, we found that steroid exposure at baseline was not significantly associated with any BID measure. Duration of steroid exposure, however, was associated with significantly higher total ASWAP scores after adjusting for multiple comparisons ( $\mathrm{p}=0.0006)$. The estimated effect of one additional year of steroid exposure on total ASWAP scores was 6.3 (1.8). Longer steroid exposure was also associated with higher Perceived Social Impact scores $(\mathrm{p}=0.002)$ and higher Subjective Dissatisfaction scores $(\mathrm{p}=0.003)$. Interestingly, however, after adding other medication usage in the propensity model for steroid use, duration of steroid exposure was no longer significantly associated with any BID measure.

The same analyses were repeated to evaluate both the baseline and cumulative impact of thiopurine and anti-TNF exposure on BID. We did not find any significant associations between BID and these classes of medications.

Disease Activity Scores: Both SCCAI and HBI scores were found to significantly decrease over time ( $\mathrm{p}=0.003$ and 0.007 , respectively). Among patients with $\mathrm{CD}$, after adjusting for age, marital status and gender, we found that all BID scores were significantly associated with disease activity as measured by HBI at baseline and follow-up wherein higher HBI scores were associated with higher total ASWAP scores $(\mathrm{p}<0.0001)$ and higher ASWAP sub-scores ( $\mathrm{p}=0.0002$ for Perceived Social Impact and $\mathrm{p}<0.0001$ for Subject Dissatisfaction) (Table 4). Among patients with UC/IBDU, we found that all BID scores were significantly associated with disease activity as measured by SCCAI at baseline and follow-up, where higher SCCAI scores were associated with higher total ASWAP scores $(\mathrm{p}=0.006)$ and higher Perceived Social Impact $(\mathrm{p}<0.0001)$ but not with greater Subjective Dissatisfaction.

HRQOL: We found IBDQ scores to be significantly associated with all BID scores among males and females wherein a 1 unit increase in the Subjective Dissatisfaction score was associated with a $0.75(0.18)$ decrease in IBDQ scores and a 1 unit increase in the Perceived Social Impact score was associated with a $1.39(0.16)$ decrease in IBDQ $(\mathrm{p}<0.0001)$ (Table 5). A one unit increase in the total ASWAP score was associated with a $0.62(0.08)$ decrease in IBDQ $(\mathrm{p}<0.0001)$. 


\section{Discussion}

The evaluation of BID in adult patients with IBD has been limited to date. Given the potential for IBD and its treatments to alter physical appearance and the association between negative body image and poor self-esteem, poor interpersonal confidence, sexual dysfunction, and mood disorders, studies examining the impact of IBD on body image are important. In this study we used a non-weight based instrument to measure BID in subjects with IBD. Changes in BID over time were assessed according to multiple factors including gender, disease location, disease activity, and medication exposure. We also examined the relationship between BID and HRQOL as measured by the IBDQ. This is the first study to our knowledge which evaluates BID among IBD patients over time.

Using a community-based, incident cohort we found that BID does not improve over time for either sex despite the fact that overall disease activity improves. Furthermore, BID remains significantly greater among females with IBD compared to males at all time points. This gender difference is likely related to the greater importance that women attach to the appearance of their bodies compared to men and the greater satisfaction that men report with their bodies compared to women in the general population. ${ }^{23,} 24$ Although work by Feingold has found differences in body satisfaction between the sexes in adulthood to be less pronounced than in adolescence ${ }^{23}$, our results suggest that gender differences in BID are significant among adult patients with IBD and that women with either disease subtype are at particular risk for the detrimental consequences of negative body image.

Our longitudinal data analysis revealed several additional interesting findings. First, after controlling for multiple potential confounders, we found no significant difference in total ASWAP scores or ASWAP sub-scores according to IBD subtype. Thus, patients with UC and $\mathrm{CD}$ appear to be equally susceptible to BID. With regards to disease location, although we saw no difference in BID scores in patients with UC according to disease location, a significant difference was found between patients with ileocolonic $\mathrm{CD}$ and colonic $\mathrm{CD}$, wherein patients with ileocolonic disease had greater BID than patients with isolated colonic disease. We postulate that differences in steroid usage between these groups may account for the difference in BID as we found steroid use at baseline to be significantly higher in patients with ileocolonic CD compared to patients with colonic involvement only (data not shown). In comparison, we found no differences in baseline steroid use among patients with UC according to disease location which may account for the stability in BID scores in this group.

We found no difference in BID among patients with $\mathrm{CD}$ according to perianal disease status. While small numbers of affected patients may have played a role in this finding, absence of a significant relationship between perianal disease and BID may be related to the relatively low importance that patients with $\mathrm{CD}$ have been found to place on the cosmetic appearance of perianal disease compared to its physical consequences. ${ }^{25}$ That is, patients may be more affected by the physical symptoms of perianal disease than its image-altering effects. In addition, we found that operative status was not associated with BID. Again, although this study may have been underpowered to find a significant association between these factors, it is important to note that several prior studies have also shown that body image scores do not 
differ significantly in patients with IBD who have undergone laparoscopic-assisted versus open surgeries despite cosmesis scores being better in the laparoscopic group, suggesting that post-operative scarring may not be a major source of BID in patients with IBD. ${ }^{26,27}$ Only 1 patient in our cohort had undergone stoma surgery by the end of the years, thus we cannot make any conclusion about the effect of stomas on BID. Prior studies have shown that presence of a stoma diminishes body image satisfaction and sexual attractiveness in patients with $\mathrm{IBD}^{7},{ }^{28}$, Longer follow-up of our cohort allowing for a greater number of surgeries is needed to further evaluate the impact of surgical therapy for IBD, particularly stoma surgery, on BID.

We found that BID was affected by symptom burden, disease activity, and certain EIM of IBD. With regards to symptom burden and disease activity, patients with higher symptom inventory scores and higher disease activity scores had greater BID. Thus, along with depression and anxiety which have been found to track with IBD activity ${ }^{29}$, BID may be an additional psychological comorbidity for which patients with active disease are at risk and for which they should be assessed. Interestingly, while HBI scores were significantly associated with all 3 measures of BID in patients with $\mathrm{CD}$, we did not find an association between Subjective Dissatisfaction scores and SCCAI scores in patients with UC. This may be due to differences in the degree and the nature of the physical appearance changes which occur in patients with active CD versus active UC. That is, patients with $\mathrm{CD}$ may experience more physical changes during periods of disease activity, perhaps related to malabsorption and weight loss, as compared to patients with UC, leading to greater subjective dissatisfaction with their appearance.

Regarding EIMs, we found that dermatologic and musculoskeletal manifestations of IBD were associated with greater BID. Given that the two most common IBD-associated dermatoses, erythema nodosum (EN) and pyoderma gangrenosum (PG), present in areas which can be kept "hidden" if so desired by clothing, it is notable that not only were Subjective Dissatisfaction scores greater in patients with these conditions so were Perceived Social Impact scores. This suggests that patients with IBD-related dermatoses perceive increased stigmatization due to their physical appearance even when they have the ability to keep their skin lesions out of public sight. Prior researchers have noted that mild disfigurements can cause equal or even greater anxiety than highly visible conditions. ${ }^{30}$ This may be due to the development of less consistent and effective coping mechanisms in patients with less noticeable physical changes such as patients with EN or PG whose lesions can be covered. ${ }^{31}$

Why the musculoskeletal manifestations of IBD are associated with greater BID is less clear given that most do not produce significant physical disfigurement. Differences in systemic steroid use for symptom management may provide some explanation and explain why patients with ocular manifestations who are typically treated with local therapy do not experience increased BID. Our medication analysis revealed that of the major classes of medications used to treat IBD only steroid use was significantly associated with BID. Univariate analysis found steroid use to be associated with all measures of BID. However, after controlling for multiple potential confounders including propensity for steroid usage, we found steroid use was significantly associated with higher Perceived Social Impact 
scores only. We, therefore, propose that the dissatisfaction in body image experienced by IBD patients treated with steroids is more related to how they perceive others view them rather than to how they view themselves.

In addition, we found that the greater the duration of steroid exposure, the greater the BID that patients experience. Interestingly, this relationship was found to be modified by the concurrent use of steroid-sparing agents as when other medication use was added to our model, duration of steroid exposure was no longer significantly associated with BID. Steroid-sparing medications such as thiopurines and anti-TNF agents, therefore, appear to have the beneficial effect of reducing steroid-associated BID, possibly by lowering the dose of corticosteroid needed to manage symptoms. They also, notably, were not found to be independently associated with greater BID despite the potential side effects which can change physical appearance which are associated with both classes of medication.

Finally, we found greater BID to be significantly associated with lower IBDQ scores suggesting that body image plays a role in HRQOL in patient with IBD Interventions aimed at improving body image satisfaction, therefore, may be an appropriate target for improving HRQOL in this patient population.

This study provides important insight on BID in patients with newly diagnosed IBD. It is limited, however, by several factors. First, the ASWAP tool, although validated to measure BID in a population with a chronic medical illness, has not been validated in patients with IBD. It, therefore, may not have been the optimal measure for BID in this cohort. We do, however, consider it to be a reasonable exploratory tool as unlike the majority of measures of body image it is constructed to measure non-weight related BID. Furthermore, others groups have used modified versions of the SWAP to evaluate patient populations who are subject to lifelong changes in appearance due to their disease and have reported meaningful

results. ${ }^{32}$-up studies using this tool or the recently published body image scale are needed to confirm our findings and compare the magnitude of our findings to other IBD populations. ${ }^{28}$ Second, few patients in our cohort had perianal disease or had undergone surgery by the end of follow-up. This study was therefore underpowered to determine whether these are risk factors for increased BID. Further follow-up of our cohort is needed to ascertain this. Finally, as our study did not include a healthy control group we are unable to determine the magnitude of body image impairment associated with a diagnosis of IBD in comparison to a healthy population.

\section{Conclusions}

In summary, using a community-based incident cohort we found that BID in patients with IBD remains stable over time despite improvements in disease activity. At all time points BID remains greater for females compared to males and is associated with lower HRQOL. Symptom burden, disease activity, dermatologic and musculoskeletal manifestations of IBD, and ileocolonic disease location for patients with $\mathrm{CD}$ were associated with greater BID while IBD subtype, ocular manifestations, and disease location among patients with UC were not. Longer follow-up of the cohort, allowing for a greater number of cases of perianal disease and IBD surgeries is needed to understand the relationship between these aspects of 
the disease and body image. Of the major medication classes used to treat IBD only steroid use was associated with BID. Importantly, use of steroid-sparing medications was found to modify the detrimental effects of prolonged steroid use on body image satisfaction.

Providers caring for patients with IBD should be aware of the risk factors for BID in this patient population given its impact on HRQOL as well as the potential for thiopurines and anti-TNFs to reduce the BID associated with steroid use.

\section{Acknowledgments}

Grant Support: This project was supported by a grant from the CCFA through the Centers for Disease Control and Prevention (1 UO1 DP000340-03) and the National Institutes of Health (1R21DK078555-01). Sumona Saha, M.D. was supported by Award Number K12HD055894 from the National Institute of Child Health and Human Development (NICHD). The findings and conclusions in this report are those of the authors and do not necessarily represent the official position of the Centers for Disease Control and Prevention or the NICHD.

\section{Appendix. Modified Adapted Satisfaction With Appearance Scale*}

Using the following scale, please state your level of agreement with the statements below

\begin{tabular}{|c|c|c|c|c|c|c|}
\hline $\mathbf{1}$ & $\mathbf{2}$ & $\mathbf{3}$ & $\mathbf{4}$ & $\mathbf{5}$ & $\mathbf{6}$ & $\mathbf{7}$ \\
\hline Strongly disagree & Disagree & Somewhat disagree & Neutral & Somewhat agree & Agree & Strongly agree \\
\hline
\end{tabular}

1. Because of changes in my appearance caused by my illness, I am uncomfortable in the presence of my family.

2. Because of changes in my appearance caused by my illness, I am uncomfortable in the presence of my friends.

3. Because of changes in my appearance caused by my illness, I am uncomfortable in the presence of strangers

4. My appearance makes other people feel uncomfortable

5. Changes in my appearance have interfered with my relationships

6. I feel that my appearance is unattractive to others

7. I don't think people would want to touch me

8. I am satisfied with the appearance of my face.

9. I am satisfied with the appearance of my neck.

10. I am satisfied with the appearance of my hands

11. I am satisfied with the appearance of my arms

12. I am satisfied with the appearance of my legs.

13. I am satisfied with the appearance of my chest.

14. I am satisfied with the appearance of my abdomen

15. I am satisfied with my overall appearance.

*This survey has been adapted from Heinberg et al. [18].

\section{References}

1. Cash TF. Body image: past, present, and future. Body Image. 2004; 1:1-5. [PubMed: 18089136]

2. Smolak, L.; Thompson, JK. Body image, eating disorders, and obesity in children and adolescents: Introduction to the second edition. In: Smolak, L.; Thompson, JK., editors. Body image, eating 
disorders, and obesity in youth. American Psychological Association; Washington, DC: 2009. p. 3-14.

3. Pinquart M. Body image of children and adolescents with chronic illness: A meta-analytic comparison with healthy peers. Body Image. 2013; 10:141-148. [PubMed: 23219705]

4. Rosenström T, Jokela M, Hintsanen M, et al. Body-image dissatisfaction is strongly associated with chronic dysphoria. J Affect Disord. 2013 Apr 29.

5. Pujols Y, Cindy MM, Seal Brooke N. The association between sexual satisfaction and body image in women. J Sex Med. 2010; 7:905-16. [PubMed: 19968771]

6. Rumsey N, Clarke A, Musa M. Altered body image: the psychosocial needs of patients. Br J Community Nursing. 2002; 7:563-566.

7. Muller K, Prosser R, Bampton P, et al. Female gender and surgery impair relationships, body image, and sexuality in inflammatory bowel disease: Patient perceptions. Inflamm Bowel Dis. 2010; 16:657-63. [PubMed: 19714755]

8. Patel KV, Farrant P, Sanderson JD, et al. Hair loss in patients with inflammatory bowel disease. Inflamm Bowel Dis. 2013; 19:1753-63. [PubMed: 23624889]

9. Trost LB, McDonnell JK. Important cutaneous manifestations of inflammatory bowel disease. Postgrad Med J. 2005; 81:580-585. [PubMed: 16143688]

10. Sarnes E, Crofford L, Watson M, et al. Incidence and US costs of corticosteroid-associated adverse events: a systematic literature review. Clin Ther. 2011; 33:1413-32. [PubMed: 21999885]

11. Perrett CM, Walker SL, O'Donovan P, et al. Azathioprine treatment photosensitizes human skin to ultraviolet A radiation. Br J Dermatol. 2008; 159:198-204. [PubMed: 18489587]

12. Tosti A, Pazzaglia M. Drug reactions affecting hair: diagnosis. Dermatologic Clinics. 2007; 25:223-31. [PubMed: 17430759]

13. Gullin G, Kroshinsky D, Cheifetz AS, et al. Psoriasis associated with anti-tumour necrosis factor therapy in inflammatory bowel disease: a new series and a review of 120 cases from the literature. Aliment Pharmacol Therapeut. 2011; 34:1318-27.

14. Harvey RF, Bradshaw JM. A simple index of Crohn's disease activity. Lancet. 1980; 315:11341135. [PubMed: 6103463]

15. Walmsley RS, Ayres R, Pounder CS, et al. A simple clinical colitis activity index. Gut. 1998; 43:29-32. [PubMed: 9771402]

16. Guyatt G, Mitchell A, Irvine EJ, et al. A new measure of health status for clinical trials in inflammatory bowel disease. Gastroenterology. 1989; 96:804-10. [PubMed: 2644154]

17. Satsangi J, Silverberg MS, Vermeire S, et al. The Montreal classification of inflammatory bowel disease: controversies, consensus, and implications. Gut. 2006; 55:749-753. [PubMed: 16698746]

18. Heinberg LJ, Kudel I, White B, et al. Assessing body image in patients with systemic sclerosis (scleroderma): validation of the adapted satisfaction with appearance scale. Body Image. 2007; 4:79-88. [PubMed: 18089254]

19. Lawrence JW, Heinberg LJ, Roca R, et al. Development and validation of the Satisfaction With Appearance Scale: Assessing body image among burn-injured patients. Psychological Assessment. 1998; 10:64-70.

20. Robins JM, Hernan MA, Brumback B. Marginal structural models and causal inference in epidemiology. Epidemiology. 2000; 11:550-560. [PubMed: 10955408]

21. Hernán MÁ B, Brumback B, Robins JM. Marginal Structural Models to Estimate the Causal Effect of Zidovudine on the Survival of HIV-Positive Men. Epidemiology. 2000; 11:561-70. 2000. [PubMed: 10955409]

22. Little, RJA.; Rubin, DB. Statistical Analysis with Missing Data. 2nd. New York: John Wiley; 2002. Oberg P, Tornstam L. Body images among men and women of different ages. Ageing Soc. 1999; 19:629-644.

23. Feingold A, Mazzella R. Gender Differences in Body Image Are Increasing. Psychological Sciences. 1998; 9:190-198.

24. Mahadev S, Young JM, Selby W, et al. Quality of Life in Perianal Crohn's Disease: What Do Patients Consider Important? Dis Colon Rectum. 2011; 51:579-585. [PubMed: 21471759] 
25. Eshuis EJ, Polle SW, Slors JF, et al. Long-Term Surgical Recurrence, Morbidity, Quality of Life, and Body Image of Laparoscopic-Assisted vs. Open Ileocolic Resection for Crohn's Disease: A Comparative Study. Dis Colon Rectum. 2008; 51(6):858-867. [PubMed: 18266036]

26. Dunker MS, Bemelaman WA, Slors JFM, et al. Functional outcome, quality of life, body image, and cosmesis in patients after laparoscopic-assisted and conventional restorative proctocolectomy. Dis Colon Rectum. 2001; 44:1800-7. [PubMed: 11742165]

27. Casati J, Toner BB, de Rooy EC, et al. Concerns of patients with inflammatory bowel disease: a review of emerging themes. Dig Dis Sci. 2000 Jan; 45(1):26-31. [PubMed: 10695609]

28. McDermott E, Moloney J, Rafter N, et al. The body image scale: a simple and valid tool for assessing body image dissatisfaction in inflammatory bowel disease. Inflamm Bowel Dis. 2014; 20:286-90. [PubMed: 24374873]

29. Porcell P, Leoci C, Guerra V. A prospective study of the relationship between disease activity and pyschologic distress in patients with inflammatory bowel disease. Scand J Gastroenterol. 1996; 31:792-6. [PubMed: 8858749]

30. Macgregor, F. After plastic surgery: Adaptation and adjustment. New York: Praeger; 1979.

31. Rumsey N, Harcourt D. Body image and disfigurement: issues and interventions. Body Image. 2004; 1:83-97. [PubMed: 18089143]

32. Franzblau L, Chung KC. Pyschosocial outcomes and coping after complete avulsion traumatic brachial plexus injury. Disabil Rehabil. 2014 Epub. 


\section{Demographic information}

\begin{tabular}{|c|c|c|}
\hline \multicolumn{2}{|l|}{ Characteristic } & \multirow{2}{*}{$\begin{array}{c}\mathbf{N}(\%) \\
91(33.2)\end{array}$} \\
\hline Age at diagnosis & $18-29$ & \\
\hline & $30-44$ & $73(26.6)$ \\
\hline & $45-65$ & $88(32.1)$ \\
\hline & $>65$ & $22(8.0)$ \\
\hline \multicolumn{2}{|l|}{ Female } & $169(62)$ \\
\hline BMI & $<18.5$ & $95(34.7)$ \\
\hline \multirow[t]{3}{*}{ Classification } & $18.5-24.9$ & $75(27.4)$ \\
\hline & $25-29.9$ & $56(20.4)$ \\
\hline & $>30$ & $48(17.5)$ \\
\hline \multirow[t]{5}{*}{ Race } & Black or African American & $11(4.0)$ \\
\hline & White & $248(90.5)$ \\
\hline & More than one race & $4(1.5)$ \\
\hline & Other & $10(3.7)$ \\
\hline & Refused & $1(0.4)$ \\
\hline \multirow[t]{5}{*}{ Marital Status } & Married & $102(37.2)$ \\
\hline & Single/Never married & $115(42.0)$ \\
\hline & Divorced or Separated & $34(5.1)$ \\
\hline & Cohabitating & $8(2.92)$ \\
\hline & Widowed & $1(0.36)$ \\
\hline
\end{tabular}

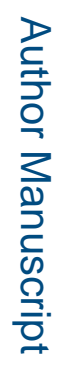

Abbr: BMI=body mass index

\section{Table 1}


Table 2

Disease characteristics and patient history at baseline

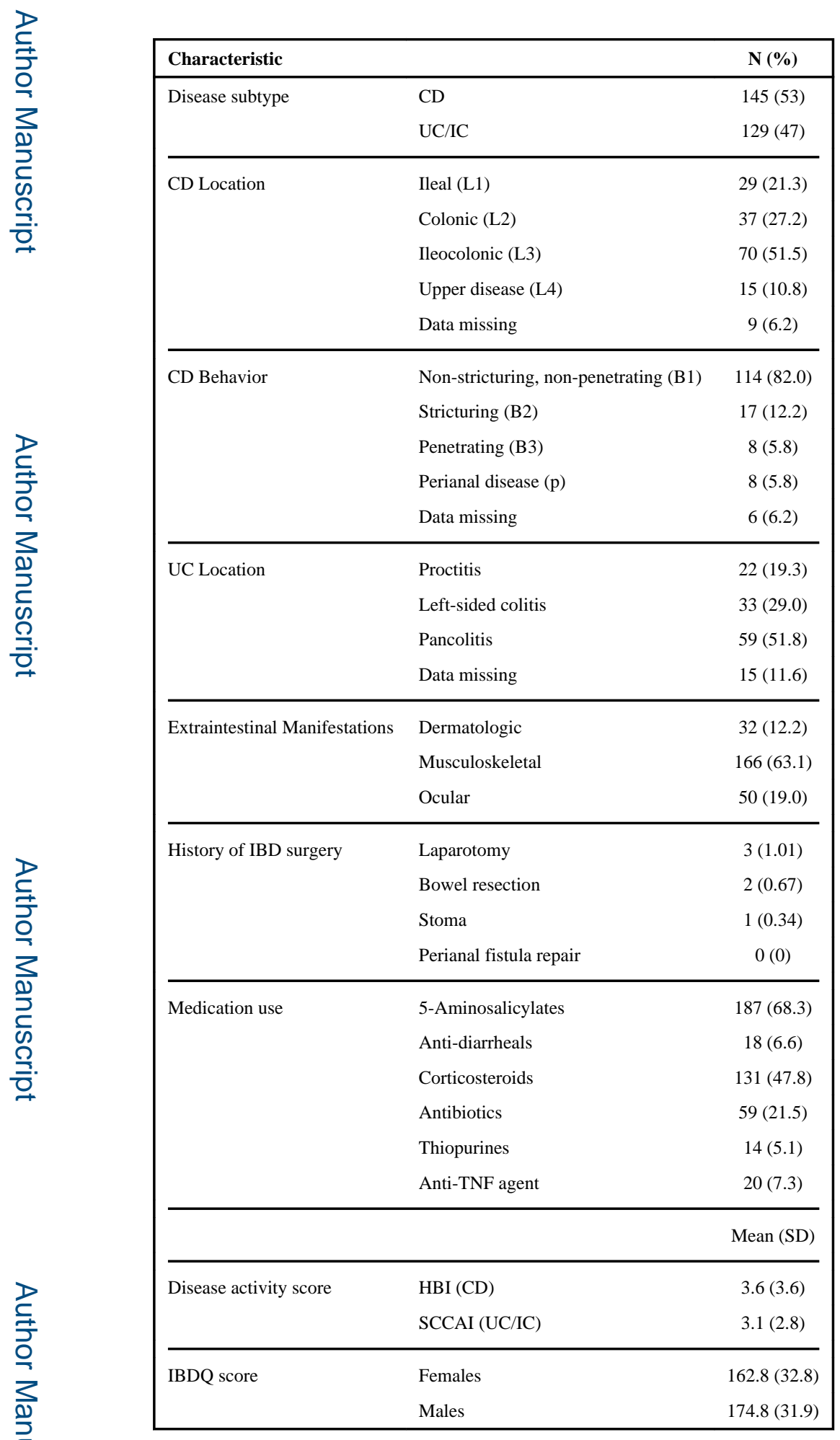

Inflamm Bowel Dis. Author manuscript; available in PMC 2016 February 01. 
Abbr: $\mathrm{CD}=$ Crohn's disease, $\mathrm{UC}=$ ulcerative colitis, $\mathrm{IC}=$ indeterminate colitis, $\mathrm{HBI}=$ Harvey-Bradshaw Index, $\mathrm{SCCAI=Simple} \mathrm{Clinical}$ Colitis Activity Index 


\section{Modified ASWAP scores}

\section{Table 3}

\begin{tabular}{|l|c|c|c|}
\hline \multirow{2}{*}{ Score } & \multicolumn{2}{|c|}{ Mean Score (SD) } & \multirow{2}{*}{ p-value* } \\
\cline { 2 - 3 } & Females & Males & \multirow{2}{*}{$<0.0001$} \\
\hline Total Score & \\
\hline Baseline & $30.1(14.4)$ & $21.2(8.4)$ & \\
\hline Year 1 & $28.2(14.1)$ & $24.5(12.5)$ & \multirow{2}{*}{$<0.0001$} \\
\hline Year 2 & $28.8(13.2)$ & $24.1(13.5)$ & \\
\hline Perceived Social Impact* & \\
\hline Baseline & $11.5(7.7)$ & $8.3(3.8)$ & \\
\hline Year 1 & $10.8(7.8)$ & $10.0(6.7)$ & \\
\hline Year 2 & $10.5(7.0)$ & $10.2(6.7)$ & \\
\hline Subjective Dissatisfaction Scale* & \\
\hline Baseline & $18.5(8.7)$ & $12.9(6.1)$ & \multirow{2}{*}{$<0.0001$} \\
\hline Year 1 & $17.9(8.2)$ & $14.6(6.9)$ & \\
\hline Year 2 & $18.3(8.5)$ & $13.9(7.4)$ & \\
\hline
\end{tabular}

p-value $<0.0001$ for gender, but insignificant for year. 


\section{Factors associated with BID}

Table 4

\begin{tabular}{|c|c|c|c|c|}
\hline Outcome & Variable & Estimate & Unadjusted p-value & Adjusted p-value \\
\hline \multirow{14}{*}{ Total modified ASWAP score } & Year & 0.75 & 0.15 & 1 \\
\hline & Gender & -6.78 & $<0.0001$ & $<0.001$ \\
\hline & IBD subtype & -0.69 & 0.64 & 1 \\
\hline & Steroids use & 2.24 & 0.03 & 0.3 \\
\hline & Symptom Inventory Score & 0.38 & $<0.0001$ & $<0.001$ \\
\hline & BMI & 0.26 & 0.008 & 0.08 \\
\hline & HBI score & 0.85 & $<0.0001$ & $<0.001$ \\
\hline & SCCAI score & 0.88 & 0.003 & 0.03 \\
\hline & CD Location: L1 vs. L3 & -3.33 & 0.26 & 1 \\
\hline & CD Location: L2 vs. L3 & -5.99 & 0.03 & 0.3 \\
\hline & Perianal disease & 2.79 & 0.56 & 1 \\
\hline & UC Location: E1 vs. E3 & 1.28 & 0.66 & 1 \\
\hline & UC Location:E2 vs. E3 & -0.07 & 0.98 & 1 \\
\hline & EIM & 4.15 & 0.0002 & 0.002 \\
\hline \multirow{14}{*}{ Perceived Social Impact Scale score } & Year & 0.13 & 0.61 & 1 \\
\hline & Gender & -2.12 & 0.01 & 0.1 \\
\hline & IBD subtype & -1.02 & 0.18 & 1 \\
\hline & Steroids use & 1.54 & 0.004 & 0.04 \\
\hline & Symptom Inventory Score & 0.19 & $<0.0001$ & $<0.001$ \\
\hline & BMI & 0.09 & 0.06 & 0.6 \\
\hline & HBI score & 0.42 & 0.0006 & 0.006 \\
\hline & SCCAI score & 0.54 & $<0.0001$ & $<0.001$ \\
\hline & CD Location: L1 vs. L3 & -1.57 & 0.28 & 1 \\
\hline & CD Location: L2 vs. L3 & -3.66 & 0.009 & 0.09 \\
\hline & Perianal disease & 1.38 & 0.56 & 1 \\
\hline & UC Location: E1 vs. E3 & 1.00 & 0.50 & 1 \\
\hline & UC Location:E2 vs. E3 & -0.42 & 0.75 & 1 \\
\hline & EIM & 1.96 & 0.002 & 0.02 \\
\hline \multirow{9}{*}{ Subjective Dissatisfaction Scale score } & Year & 0.58 & 0.08 & 0.8 \\
\hline & Gender & -4.58 & $<0.0001$ & $<0.001$ \\
\hline & IBD subtype & 0.39 & 0.66 & 1 \\
\hline & Steroids use & 0.81 & 0.21 & 1 \\
\hline & Symptom Inventory Score & 0.22 & $<0.0001$ & $<0.001$ \\
\hline & BMI & 0.16 & 0.007 & 0.07 \\
\hline & HBI score & 0.49 & $<0.0001$ & $<0.001$ \\
\hline & SCCAI score & 0.33 & 0.11 & 1 \\
\hline & CD Location: L1 vs. L3 & -1.74 & 0.30 & 1 \\
\hline
\end{tabular}




\begin{tabular}{|l|l|c|c|c|}
\hline Outcome & Variable & Estimate & Unadjusted p-value & Adjusted p-value \\
\hline \multirow{4}{*}{} & CD Location: L2 vs. L3 & -2.26 & 0.15 & 1 \\
\cline { 2 - 5 } & Perianal disease & 2.11 & 0.43 & 1 \\
\cline { 2 - 5 } & UC Location: E1 vs. E3 & 0.73 & 0.69 & 1 \\
\cline { 2 - 5 } & UC Location:E2 vs. E3 & 0.25 & 0.87 & 1 \\
\cline { 2 - 5 } & EIM & 2.92 & $<0.0001$ & $<0.001$ \\
\hline
\end{tabular}

Abbr: BMI=body mass index, HBI=Harvey-Bradshaw Index, SCCAI=Simple Clinical Colitis Activity Index, CD=Crohn's disease, UC=ulcerative colitis, EIM=extraintestinal manifestation

* Adjusted for age, gender, and marital status 
Table 5

\section{Association between BID measures and HRQOL}

\begin{tabular}{|l|l|c|c|c|}
\hline Outcome & Variable & Estimate & Unadjusted p-value & Adjusted p-value \\
\hline \multirow{4}{*}{ IBDQ } & Perceived Social Impact & -2.10 & $<0.0001$ & $<0.001$ \\
\cline { 2 - 5 } & Subjective Dissatisfaction Scale & -1.52 & $<0.0001$ & $<0.001$ \\
\cline { 2 - 5 } & Total modified ASWAP score & -1.17 & $<0.0001$ & $<0.001$ \\
\hline
\end{tabular}

Abbr: IBDQ=Inflammatory Bowel Disease Questionnaire 